\title{
Do DMOs Promote the Right Aspects of the Destination? A Study of Instagram Photography with a Visual Classifier
}

\author{
Lyndon Nixon ${ }^{(\bowtie)}(\mathbb{D}$ \\ School of Applied Data Science, MODUL University Vienna, Am Kahlenberg 1, \\ 1190 Vienna, Austria \\ lyndon.nixon@modul.ac.at
}

\begin{abstract}
As global travel emerges from the pandemic, pent up interest in travel will lead to consumers making their choice between global destinations. Instagram is a key source of destination inspiration. DMO marketing success on this channel relies on projecting a destination image that resonates with this target group. However, usual text-based marketing intelligence on this channel does not work as content is consumed first and foremost as a visual projection. The author has built a deep learning based visual classifier for destination image measurement from photos. In this paper, we compare projected and perceived destination images in Instagram photography for four of the most Instagrammed destinations worldwide. We find that whereas the projected destination image aligns well to the perceived image, there are specific aspects of the destinations that are of more interest to Instagrammers than reflected in the current destination marketing.
\end{abstract}

Keywords: Destination image $\cdot$ Visual classification $\cdot$ Instagram photography

\section{Introduction}

Instagram may be the most significant source of destination inspiration for a significant number of travel-interested Internet users, especially in the 18-34 year old age group ${ }^{1}$. After all, $48 \%$ of Instagram users rely on the images and videos that they see on the social media platform to inform their travel decision making and $35 \%$ of users use the platform to discover new places ${ }^{2}$. It is therefore not surprising that destination marketing organisations (DMOs) have expended significant effort in marketing on this channel. Instagram is a highly visual social network and therefore content marketing is focused on the use of photography and videos, more so than the text of postings. When an Instagram user consumes destination marketing content, the intention of the

\footnotetext{
${ }^{1} 62.8 \%$ of the users are aged 18-34 according to the Digital Internet Snapshot $2021 \mathrm{https} / / /$ datareportal.com/reports/digital-2021-global-overview-report (last accessed on 24 March 2021).

${ }^{2}$ Rezab, J. "Instagram: The Place To Be For Travel Brands". Available online. https://www.mediapost. com/publications/article/263167/instagram-the-place-to-be-for-travel-brands.html (last accessed on 28 July 2021. Requires free sign-in.).
} 
marketer is that the visually projected image of the destination resonates with them and increases their interest and positive attitude towards the destination - leading to an intent to visit the destination. Multiple case studies have backed up the assumption that an optimally marketed destination on Instagram can lead to an increase in visitor numbers $^{3}$. Indeed, Instagram is now being blamed for causing overtourism in certain "Instagrammable" locations ${ }^{4}$. A challenge for DMOs or other stakeholders is to know if they are indeed marketing their destination optimally on Instagram. A rise in visitor numbers can only suggest marketing success a posteriori. Marketing intelligence - the analysis of marketing data to identify the factors of marketing success - has classically made use of analysis of text (of reviews, or of social media postings) and quantifiable metrics (e.g., in social networks number of likes, comments and shares) [15]. However, the primary component of Instagram marketing is visual: the photo or video used in the posting. Ignoring this component in marketing intelligence in Instagram would mean the primary factor for the marketing success is being excluded from the analysis.

In this paper, our assumption is that the target audience for the destination marketing - the Instagram users - reveal the aspects they are most interested in and find most appealing in a visit to the destination through their own choice of the photography they post to the social network as part of their touristic experience. Photography has been long understood as an expression of the photographer's mental image of the destination [19]. They take photos of the aspects they consider most indicative, and this is now even more the case in social networks where the user chooses which of their photos they want to post and share with others. Therefore, the content of Instagram photography from a destination (user generated content, or UGC) can be analysed for the "perceived destination image". Equally, the content posted by the DMO on its Instagram channel can be subject to the same analysis to measure the "projected destination image" [11]. Common marketing theory says that marketers want to influence consumer attitudes towards their product and can measure the success of this influence by surveying consumers after a marketing campaign and determining if attitudes have become closer to the marketing message. In e-tourism, this has translated as marketers defining their intended "projected destination image" and measuring how close the "perceived destination image" comes to it after a marketing campaign. However, such thinking persists the assumption that marketers are the primary conveyors of the destinations image when in fact today's traveller has a destination image which is strongly influenced by the UGC they consume about that destination [14], especially from social networks [25]. Therefore, we turn this theory on its head and argue that, in fact, destination marketers should inform themselves of the "perceived destination image" of their target audience and, given that this reflects the that resonate most with those consumers, aim to align their "projected destination image" to it.

Measuring destination image from Instagram photography is quite different from analysis of text or statistics. In the following chapter, we look at destination image in e-

\footnotetext{
3 e.g. Iceland and New Zealand case studies from "Insta tourism explained: What, why and where". Available online. https://tourismteacher.com/insta-tourism/ (last accessed on 28 July 2021).

4 Tenbarge, K. "15 destinations Instagram has helped ruin” Available online. https://www.insider.com/ travel-destinations-instagram-influencers-ruined-2019-7 (last accessed on 28 July 2021).
} 
tourism research and how visual media classification has been researched in the field of computing. Section 3 introduces the author's implementation of a deep learning-based visual classifier for measuring destination image and its evaluation results for accurate classification of tourism photography. Section 4 explains our experiment: we take four top Instagram destinations and download sets of DMO and UGC photos. We describe the measured destination image as multidimensional vectors and compare projected and perceived images including for selected features. We identify differences between the destination marketing and UGC. Section 5 concludes this paper with a look at our findings, what DMOs can learn from this for their future destination marketing and which limitations and future work remain.

\section{State of the Art}

Destination image has been a subject of tourism research since several decades [5]. It has been defined as the mental construct a person has of a destination and is sourced initially from indirect sources, i.e., before they visit the destination and form an opinion [8]. From the perspective of a group, the destination image may be considered the ideas and feelings most expressed about the destination (e.g., Costa Rica may be most typically associated with jungle and adventure). Destination image has been important as a concept to tourism stakeholders as a correlation is assumed between a positive image and an increased intention to visit [16]. Originally, destination marketers considered their marketing as significant in determining the image of their destination among a target audience, as their marketing materials were a primary source of impressions about the destination for someone who was exploring the option to visit. Nowadays this no longer holds as the Web and digital photography has led to an explosion of destination imagery from tourists which is globally distributed and easily consumed on popular Websites. This imagery is now dominant in forming an initial destination image among connected consumers [13]. Destination marketers now need to accept that Internet users participate in the co-creation of the destination brand [1], referring to the influence on the destination image of the user content being shared online [2].

Initial destination image research used either surveys and other solicitation techniques from the public or expert knowledge to define the attributes people commonly associate with destinations [8]. Measurement of destination image consists of determining a value for each attribute. Given a classification of those attributes, surveys were also used to directly extract the destination image for a given destination from a group of people, e.g. [3]. As more destination related content became available online through Websites, researchers turned from survey-based approaches to data-based approaches where they collected the data from the Web and used software tools to analyse it. Text analysis could be used with collections of reviews of tourism POIs (points of interest), e.g. [15]. However, this proves less effective with multimedia content (images and videos) where the only text that might be available would be an associated description, tags or even the content of the comments on that multimedia item. 
Tourism researchers have long been aware of the importance of photography in understanding how visitors perceive a destination [6]. The choice of elements in the photos are seen as reflecting the photographer's own idea of what is most important about the destination [19]. Initial research on destination image and photography solicited directly from participants their impressions from observing a certain photo, a technique known as PEI (Photo Elicitation Interview), which has also been applied with Instagram [9]. Later work manually classified the photos found on Websites according to their content or theme, e.g. [21, 22]. Such efforts were largely small scale and did not concur in how to classify the photos, nor aligned their classifications to other research on destination image attributes [20].

With the advent of social networks and photo sharing, the scale of available photography about destinations has expanded greatly. The Web also drove "big data" research where analysis could be performed on larger scale data collections that could be sourced online and hence provide more accurate results. However, in the research domain of image understanding, accessible and accurate software systems have only emerged with the advent of deep learning-based approaches [24]. These use neural networks (AI systems that seek to replicate the neural activity of the brain) with multiple processing layers (hence "deep") to 'learn' to identify the content of images based on training with large datasets of already classified images. State of the art deep learning classifiers have been trained with the ImageNet dataset, a set of 1.3 million photos annotated according to a list of 1000 visual categories, reporting high levels of accuracy when evaluated against previously unseen image sets ${ }^{5}$. The classification abilities of deep neural networks are also available to users via Web based APIs hosted by companies such as Google (Cloud Vision), IBM (Watson Visual Recognition) or Microsoft (Azure Computer Vision). They tend to offer object recognition, i.e., classification of images based on the detection of visible objects, drawing from concept sets in the tens of thousands.

Tourism research using deep learning classifiers to analyse larger photo sets has only appeared in the past few years, cf. [7, 12, 26-28]. Off-the-shelf pre-trained classifiers have been used which return generic concepts for each image, largely based on the ImageNet classification. A few papers have acknowledged that this is not directly useable in the touristic context where destination image is defined as an aggregation of more general cognitive attributes (such as "entertainment") rather than the specific objects explicitly visible in the image (such as "guitar", "turntables" or "stage"). The classifiers are not trained specifically on tourist photography and the few experiments to measure the accuracy of a classifier when used in the tourism domain report lower figures than for the ImageNet classification for which evaluation results are reported. [18] evaluated two pre-trained classifiers with Instagram photography of Vienna and found f1 measures (the harmonic mean of precision and recall) of 0.54 . [12] found an accuracy score of $28 \%$ for their classifier when tested specifically in the use case of touristic destination image. Past work has also not mapped the labels

\footnotetext{
5 https://keras.io/api/applications/ lists pre-trained models available in the Python library Keras with top-5 accuracy scores of 89 to $96 \%$.
} 
returned by the off-the-shelf classifiers to destination image attributes, a task made more complex by the lack of any official listing of all labels.

There is the option to train a deep learning neural network specifically for visual classification in some new domain, given that the ImageNet pre-training is accepted to be too generic when classification is to be done for a specific use case. While networks could be trained from scratch, transfer learning is a common approach to reach high accuracy with less training cycles. Here, we start with the pre-trained model from ImageNet and then train the new model with the domain-specific annotated images. However, to the best of the author's knowledge, no-one has trained a visual classifier specifically for destination image measurement. In the next chapter, we introduce the author's implementation and evaluation of such a system.

\section{Implementation of a Visual Classifier for Destination Image}

Given the lack of an accurate classifier for destination image from photography, we decided to implement our own. While the leading benchmark systems in (general) visual classification tend to be highly complex (hundreds of layers and ten of millions of parameters) they also need expensive, powerful hardware to run. In our case, we are considering a more specific task (narrow domain) and aim for a model which can be trained and used with more common computing resources (e.g., a laptop with one GPU, or in cloud services such as Google Colab). We develop in Python, using the Keras open-source library for developing deep learning networks.

The standard workflow for a deep learning model is to prepare training data, build a pipeline to input the data, build a model, train the model with the data, test the model and then iterate on improving the model. To prepare training data, we need to decide on the set of visual categories for which the classifier will be trained then prepare a set of data for training which consists of tourism photography where each photo is annotated as belonging to one of those visual categories (every photo is considered as belonging to a single category. Multi-label classification is a subject for future work). Finally, train a model with the data and measure the accuracy on the test data set (we follow a standard convention of taking $20 \%$ of the data for testing).

To choose the visual categories for the classifier, we want to use the commonly accepted cognitive attributes of destination image as defined in the research literature. However, there is no single, widely accepted definition of destination image attributes. We start with the factors influencing destination image defined by [4] as the authors aimed for a comprehensive aggregation of all attributes that are considered in a destination. [17] wanted to measure destination image from Instagram photography (through a survey) and took a subset of the list in [4] which was best suited to cognitive identification (tangibly visible in an image). This led to a new list of 53 attributes where some attributes were more specific than [4] (e.g., "Flora and Fauna" became "Plants \& Flowers", "Animals" and "Trees"). In a further refinement step, the author took this list of 53 attributes and cleaned it to 18 visually distinct categories based on aggregations (e.g., "Concerts" and "Cinema/Theatre" could be grouped into "Entertainment") and filtering out categories that are difficult to generalise for classification (e.g., "Arts and Crafts" and "Traditions" would look very different from place to place) (Table 1): 
Table 1. Summary of [4]'s destination image attributes following aggregation and filtering by the author into visually distinct categories

\begin{tabular}{|c|c|c|}
\hline Natural Resources & General Infrastructure & Tourist Infrastructure \\
\hline $\begin{array}{l}\text { Beaches } \\
\text { Landscape } \\
\text { Water } \\
\text { Mountains } \\
\text { Desert } \\
\text { Plants and flowers } \\
\text { Animals } \\
\text { Trees }\end{array}$ & Roads and traffic & $\begin{array}{l}\text { Shops and markets } \\
\text { Accommodations } \\
\text { Gastronomy }\end{array}$ \\
\hline Leisure and recreation & Culture, history and art & \\
\hline $\begin{array}{l}\text { Sport } \\
\text { Entertainment }\end{array}$ & $\begin{array}{l}\text { Art and museums } \\
\text { Historical buildings } \\
\text { Modern building } \\
\text { Monuments }\end{array}$ & \\
\hline
\end{tabular}

To create the training data, we have noted that available classifiers have used public large scale annotated photo datasets such as ImageNet. However, these datasets are not annotated with tourism photography and destination image in mind and tend towards generic object detection tasks such as identifying a "car", "dog" or "football". Therefore, we found it important to source a new image training dataset specifically for our task. There is no golden rule for how many images are needed for training each category but following the TensorFlow tutorial for visual classification ${ }^{6}$ where 3670 photos were used to train for 5 classes i.e., 734 photos per class, we also aimed to average at several hundred photos for each category. To find photos suitably representative of tourism photography in each category, we used Google Images search with the conjunctive query "tourism AND (label)" where label is the name of the visual category (categories like "plants \& flowers" were split into two queries). The Fatkun Batch Bild plugin was used to download 500-800 images per category and the result was manually filtered to colour photographs without any overlays, leading to $100-500$ photos per category for the training dataset. The final training dataset has 4949 photos, or an average of 275 photos per category. After training, the photos were discarded.

The initial architecture is a Convolutional Neural Network (CNN) with three convolution blocks, a max pooling layer in each, a fully connected layer on top with 128 units activated by a relu activation function and, in our case another fully connected layer with 18 units which outputs the result (a confidence value for each of the 18 visual categories). While the initial CNN had an accuracy score of $63-68 \%$ on the test dataset (after 20 epochs), we decided to explore the use of transfer learning to train our new model on top of the visual classification already learnt by an existing, re-usable model. Several models are available in Keras for transfer learning and after experimenting with results from a few options, we chose InceptionResNetV2 which reports

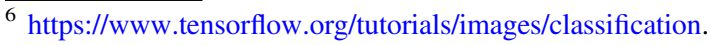


the best results on the ImageNet validation dataset yet is not the most complex to train with (56 million parameters) ${ }^{7}$. Further experimentation with the settings for the model training led us to choose max pooling and making all layers trainable. We loaded InceptionResNetV2 without the last two convolutional layers and added three convolution blocks with a max pooling layer in each, as in our original CNN. We run this new model for 10 epochs, and this model scored $90 \%$ accuracy on the test dataset.

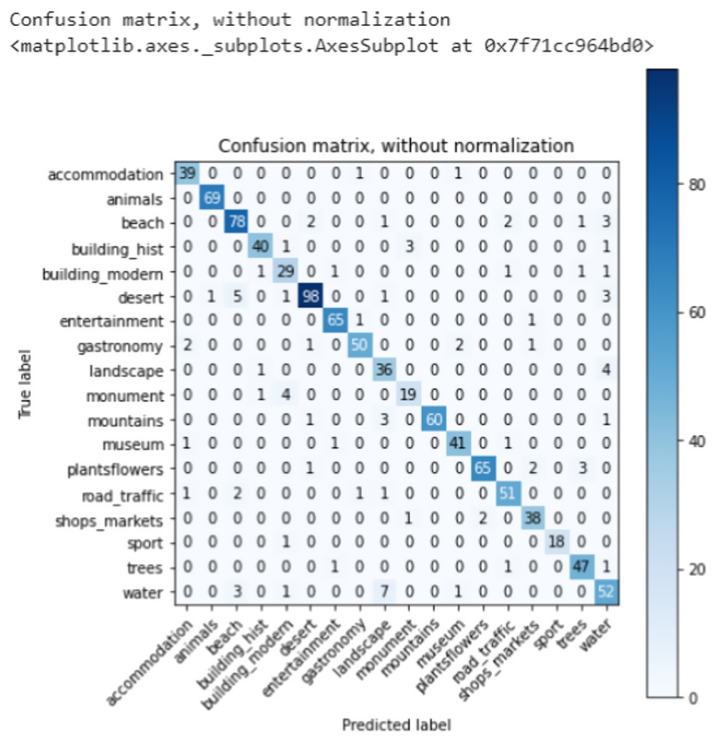

Fig. 1. Confusion matrix for classification of the test dataset

We produced a confusion matrix to check if the accuracy is consistent across the categories (Fig. 1. NB the 18 visual categories can be seen on the vertical axis in the order they are classified). In the test dataset (983 images), 29 out of 34 images (85\%) labelled building_modern and 19 out of 24 images (79\%) labelled monument were predicted correctly with the main confusion being 4 monument images labelled as building_modern (out of 24). Other confusions visible in the matrix are desert images labelled as beach (5), landscape as water (4) and water as landscape (7), all of which are understandable given the common-sense similarities (deserts and beaches both have sand, landscapes also contain water). Overall, the classifier has correctly labelled the vast majority of images and has been consistent across categories.

\footnotetext{
${ }^{7}$ InceptionResNetV2 Simple Introduction, by Zahra Elhamraoui, Medium, May 16, 2020. https:// medium.com/@zahraelhamraoui1997/inceptionresnetv2-simple-introduction-9a2000edcdb6 (last accessed August 31, 2021).
} 


\section{Experiment}

Having made available a visual classification model that can annotate photography according to a shortlist of destination image cognitive attributes with high accuracy, we will use the classifier to compare the perceived and projected destination image of a number of top destinations according to the Instagram photos posted by users and by the DMO respectively. Based on lists of most Instagrammed locations, we chose the cities of Paris, Barcelona and New York as well as the country of the Maldives, which also complement one another in the sense of each being quite distinct as a destination (Paris: romantic, historical; Barcelona: beach, modernism; New York: urban, entertainment; Maldives: water, relaxation). We use a Python library to download Instagram photos for each destination (i) from its official DMO account and (ii) according to the DMO recommended travel hashtag (this helps focus our data collection of tourismrelated photography). We discard the photos once we have classified them. Table 2 shows the selected account and hashtag and the number of photos acquired for each (the downloading took place in the second half of August 2021):

Table 2. Photos used in the experiment by destination

\begin{tabular}{l|l|l|l|l}
\hline Destination & DMO account & No. photos & Travel hashtag & No. photos \\
\hline Maldives & @ visitmaldives & 2525 & \#visitmaldives & 1742 \\
\hline New York & @ nycgo & 1404 & \#itstimefornyc & 1017 \\
\hline Paris & @ parisjetaime & 1575 & $\begin{array}{l}\text { \#parisjetaime } \\
\text { \#jetaimeparis }\end{array}$ & 1503 \\
\hline Barcelona & @ visitbarcelona & 1675 & \#visitbarcelona & 957 \\
\hline
\end{tabular}

The classifier labels each individual photo with a set of 18 confidence scores (on a scale of 0 to 1), one for each visual category (in the order seen in Fig. 1). We accept one label per photo in that we select the visual category with the highest confidence score. Given an input of multiple photos, we can produce an array of 18 integers where each integer is the sum of photos labelled with a respective visual category. Vectors are representations of objects in multi-dimensional space. Following the use of vectors to represent data items in embedding layers of neural networks, where the items can be subsequently compared and learnt about (e.g., similar items are closer together and can be clustered), we will interpret the destination image array produced by the visual classifier as an 18-dimensional vector. Such a vector can be understood as a set of feature weights where the features are the visual categories and the weights are the presence of the feature in the dataset (to allow for comparison, all features need to be at the same scale so we take the no. of photos labelled with the feature divided by no. photos in the dataset to produce for every feature a value between 0 and 1). For example, the vector produced for the projected destination image (the DMO account) of Maldives is: $\left[\begin{array}{llllll}0.01663366 & 0.00871287 & 0.3829703 & 0.00435644\end{array}\right.$ $\begin{array}{lllll}0.02336634 & 0.02336634 & 0.02930693 & 0.02534653 & 0.02336634\end{array}$ 


\section{$\begin{array}{lllll}0.01029703 & 0.0150495 & 0.00475248 & 0.03960396 & 0.01584158\end{array}$}

$0.013465350 .010297030 .009900990 .34336634]$. The two dominant features are clearly beach (in the $3^{\text {rd }}$ position with a value of 0.383 ) and water (in the last position with a value of 0.343 ) as all other features (all other destination image attributes) are not larger than 0.04. We can say that the DMO marketing of the Maldives (projected image) is heavily based on the beach and water attributes. We can use the cosine similarity of two vectors to determine how close the projected image (DMO) is to the perceived image (UGC) of the destination. Cosine similarity measures similarity in terms of the orientation of the vectors rather than the magnitude, which makes sense for the representation of destination image in $n$-dimensional space since each weight represents a feature of the destination and the overall image is determined by the comparative relationship of the feature values (e.g., beach and water is stronger than everything else) rather than the absolute values themselves. The resulting cosine similarity measure between the Maldives' projected and perceived destination images is $\mathbf{0 . 9 8 3 ~ ( c f . ~ T a b l e ~ 3 ) , ~ i n d i c a t i n g ~ n o ~ s i g n i f i c a n t ~ d i f f e r e n c e s . ~ I t ~ s e e m s ~ M a l d i v e s ~ v i s i t o r s ~}$ equally post in their majority beach and water photos. We can consider the Maldives case a validation of our approach with the visual classifier, as the Maldives as a destination is arguably limited to the two visual categories of beach and water (it seems neither accommodation nor gastronomy form a focus of destination photography there) and therefore it could be expected that these two features dominate both the projected and perceived images of the Maldives. How about cities like New York, Paris or Barcelona, which contain many different features that could be subjects of photography? Do the DMOs present a different image of the city than the visitors themselves? Table 3 shows the cosine similarity for each destination image, i.e., the closeness of the vectors of the destinations projected destination image (DMO account) and perceived

Table 3. Cosine similarity between vectors for the projected and perceived destination image of each destination

\begin{tabular}{l|l}
\hline Maldives & 0.983 \\
\hline New York & 0.941 \\
\hline Paris & 0.946 \\
\hline Barcelona & 0.956 \\
\hline
\end{tabular}

destination image (UGC).

There is a close similarity in all cases, suggesting the projected and perceived destination images are well aligned. On one hand, DMOs can achieve this by reusing UGC in their own destination marketing, a common approach today on platforms like Instagram. On the other hand, it suggests that Instagram users do share the same perception of the destination as the one being promoted by the destination marketers a possible proof of the notion of the hermeneutic circle of representation [23]. This is the idea that we replicate and reinforce the media depictions we already know (in Paris, we have to photograph the Eiffel Tower, etc.). [10] considered that tourist photography may either reflect or inform a destination image since, consciously or unconsciously, 
tourists look for scenes that replicate their existing perceptions. While this result is positive for the DMO, there may some differences in the images which we can explore by looking at specific features (visual categories) in the destination image vector. Figure 2 shows comparisons of the city destination images along three features that all

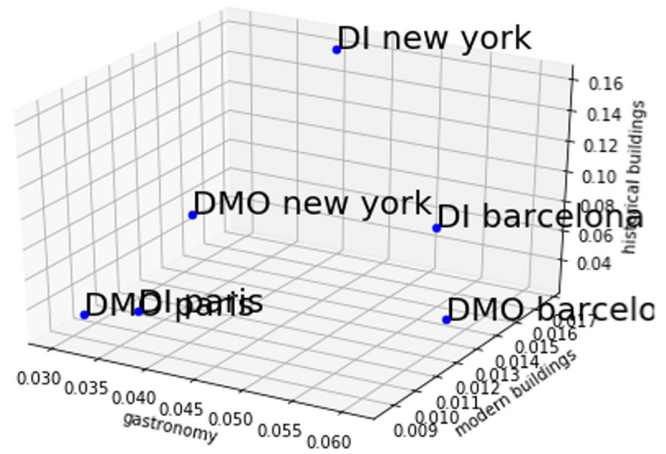

Fig. 2. $3 \mathrm{D}$ plot of the destination images of the cities along three features (DI $=$ perceived image, $\mathrm{DMO}=$ projected image)

of the cities are known for: gastronomy, historical buildings and modern buildings.

While the projected DMO imagery of Paris is closest to the perceived image in UGC (both points almost overlap), Instagrammers focus more on all three features than the DMO in New York (it may be the DMO is actively working on promoting other features of NYC) whereas in Barcelona the DMO tends to feature more gastronomy than the perceived destination image (which more strongly features both historical and modern buildings in the city). In particular, the New York DMO might be advised to focus more on gastronomy and historical buildings in NYC as this is certainly of more interest to Instagrammers, and the Barcelona DMO should consider that Instagrammers are attracted to both the historical and modern architecture of the city more than is reflected in their destination marketing.

\section{Conclusions}

In this paper, we described our own deep learning visual classifier for tourism photography which can be used to measure destination image and demonstrated its accuracy. We used this classifier with both DMO and UGC photography from Instagram for four popular destinations and considered how the resulting destination image, measured as an 18-dimensional vector, may be analysed for destination marketing insights. We found that projected and perceived images align well in Instagram, so DMOs are actively projecting an image which resonates well with Instagram users' perceptions of the destination. However, along individual features differences can be found. Assuming DMOs are well advised to align their projected images to the perceived image on Instagram, New York could promote more gastronomy and historical 
buildings and Barcelona could promote more its architecture, both historical and modern. We believe the measurement of destination image using our visual classifier can provide new insights into how destinations are presented through visual media such as photographs, which is an area of Internet marketing still not satisfactorily covered in business intelligence systems. Other experiments could be to compare the images of different destinations, to identify changes in destination images over time, or differences in destination image across different groups. As a limitation, we restricted our destination image attributes to 18 categories which were not only visually distinct but also globally consistent. Categories like arts \& crafts or traditions were not considered as their visual appearance would vary greatly from place to place. Future work would be to both further improve the accuracy of the classifier, especially considering very visually different destinations, and to add further categories (related to the previous point, possibly ad hoc based on the classification task). The work could also be applied to video, which is 'moving images', although the temporal dimension should also be considered (i.e., how long a feature is visible for). The Python code for training a CNN with transfer learning for destination image classification as well as the weights of our best performing model (90\% accuracy) are made available publicly on Github https:// github.com/lyndonnixon/VisualDestinationClassifier as well as a labelled set of Flickr photographs for evaluating visual classifiers on Google Drive https://bit.ly/ visualdestination. We hope thereby to encourage further research in visual classification for destination image and further experiments with tourism photography and videos.

\section{References}

1. Abbate T, Codini A, Aquilani B (2019) Knowledge co-creation in Open Innovation digital platforms: processes, tools and services. J Bus Ind Mark 34:1434-1447. https://doi.org/10. 1108/jbim-09-2018-0276

2. Agrawal A, Kaushik A, Rahman Z (2015) Co-creation of social value through integration of stakeholders. Procedia Soc Behav Sci 189:442-448. https://doi.org/10.1016/j.sbspro.2015. 03.198

3. Baloglu S, McCleary K (1999) A model of destination image formation. Ann Tour Res 26 (4):868-897. https://doi.org/10.1016/s0160-7383(99)00030-4

4. Beerli A, Martín JD (2004) Factors influencing destination image. Ann Tour Res 31(3):657681. https://doi.org/10.1016/j.annals.2004.01.010

5. Crompton JL (1979) Motivations for pleasure vacation. Ann Tour Res 6(4):408-424. https:// doi.org/10.1016/0160-7383(79)90004-5

6. Day J, Skidmore S, Koller T (2002) Image selection in destination positioning: a new approach. J Vacat Mark 8(2):177-186. https://doi.org/10.1177/135676670200800207

7. Deng N, Li X (2018) Feeling a destination through the "right" photos: a machine learning model for DMOs' photo selection. Tour Manag 65:267-278. https://doi.org/10.1016/j. tourman.2017.09.010

8. Echtner CM, Ritchie JB (1991) The meaning and measurement of destination image. J Tour Stud 2(2):2-12 
9. Fatanti MN, Suyadnya, IW (2015) Beyond user gaze: how instagram creates tourism destination brand? Procedia Soc Behav Sci 211:1089-1095. https://doi.org/10.1016/j.sbspro. 2015.11.145

10. Garrod B (2008) Understanding the relationship between tourism destination imagery and tourist photography. J Travel Res 47(3):346-358. https://doi.org/10.1177/00472875083 22785

11. Kim H, Richardson SL (2003) Motion picture impacts on destination image. Ann Tour Res 30(1):216-237. https://doi.org/10.1016/s0160-7383(02)00062-2

12. Kim D, Kang Y, Park Y, Kim N, Lee J (2019) Understanding tourists' urban images with geotagged photos using convolutional neural networks. Spat Inf Res 28(2):241-255. https:// doi.org/10.1007/s41324-019-00285-x

13. Marchiori E, Cantoni L (2015) The role of prior experience in the perception of a tourism destination in user-generated content. J Destin Mark Manag 4(3):194-201. https://doi.org/ 10.1016/j.jdmm.2015.06.001

14. Marine-Roig E, Anton Clavé S (2015) Tourism analytics with massive user-generated content: a case study of Barcelona. J Destin Mark Manag 4(3):162-172. https://doi.org/10. 1016/j.jdmm.2015.06.004

15. Miguéns J, Baggio R, Costa C (2008) Social media and tourism destinations: TripAdvisor case study. Adv Tour Res 26(28):1-6

16. Molinillo S, Liébana-Cabanillas F, Anaya-Sánchez R, Buhalis D (2018) DMO online platforms: image and intention to visit. Tour Manag 65:116-130. https://doi.org/10.1016/j. tourman.2017.09.021

17. Nixon L, Popova A, Önder I (2017) How instagram influences visual destination image: a case study of Jordan and Costa Rica. In: ENTER2017 conference, Rome, Italy

18. Nixon L (2018) Assessing the usefulness of online image annotation services for destination image measurement. In: ENTER2018 conference, Jönköping, Sweden

19. Pan S, Lee J, Tsai H (2014) Travel photos: Motivations, image dimensions, and affective qualities of places. Tour Manag 40:59-69. https://doi.org/10.1016/j.tourman.2013.05.007

20. Picazo P, Moreno-Gil S (2017) Analysis of the projected image of tourism destinations on photographs: a literature review to prepare for the future. J Vacat Mark 25(1):3-24. https:// doi.org/10.1177/1356766717736350

21. Stepchenkova S, Zhan F (2013) Visual destination images of Peru: comparative content analysis of DMO and user-generated photography. Tour Manag 36:590-601. https://doi.org/ 10.1016/j.tourman.2012.08.006

22. Tussyadiah P (2010) Destination-promoted and visitor-generated images - do they represent similar stories? In: Tourism and visual culture, vol 2. Method and cases, Chap. 13

23. Urry J (2002) The tourist gaze. Sage. https://doi.org/10.1079/9781845936112.0156

24. Voulodimos A, Doulamis N, Doulamis A, Protopapadakis E (2018) Deep learning for computer vision: a brief review. Comput Intell Neurosci 2018. https://doi.org/10.1155/2018/ 7068349

25. Xiang Z, Magnini VP, Fesenmaier DR (2015) Information technology and consumer behavior in travel and tourism: insights from travel planning using the internet. J Retail Consum Serv 22:244-249. https://doi.org/10.1016/j.jretconser.2014.08.005 
26. Xiao X, Fang C, Lin H (2020) Characterizing tourism destination image using photos' visual content. ISPRS Int J Geo Inf 9(12):730. https://doi.org/10.3390/ijgi9120730

27. Zhang K, Chen Y, Li C (2019) Discovering the tourists' behaviors and perceptions in a tourism destination by analyzing photos' visual content with a computer deep learning model: the case of Beijing. Tour Manag 75:595-608. https://doi.org/10.1016/j.tourman. 2019.07.002

28. Zhang K, Chen Y, Lin Z (2020) Mapping destination images and behavioral patterns from user-generated photos: a computer vision approach. Asia Pac J Tour Res 25(11):1199-1214. https://doi.org/10.1080/10941665.2020.1838586

Open Access This chapter is licensed under the terms of the Creative Commons Attribution 4.0 International License (http://creativecommons.org/licenses/by/4.0/), which permits use, sharing, adaptation, distribution and reproduction in any medium or format, as long as you give appropriate credit to the original author(s) and the source, provide a link to the Creative Commons license and indicate if changes were made.

The images or other third party material in this chapter are included in the chapter's Creative Commons license, unless indicated otherwise in a credit line to the material. If material is not included in the chapter's Creative Commons license and your intended use is not permitted by statutory regulation or exceeds the permitted use, you will need to obtain permission directly from the copyright holder. 